\title{
Reverse transcription PCR to detect low density malaria
}

\section{infections [version 1; peer review: 2 approved with}

\section{reservations, 1 not approved]}

\section{Peter Christensen (D1,2, Zbynek Bozdech³, Wanitda Watthanaworawit ${ }^{1}$, Laurent Rénia4,5, Benoît Malleret (iD4,6, Clare Ling 1,7, François Nosten (iD1,7}

\author{
${ }^{1}$ Shoklo Malaria Research Unit, Mahidol University, Maesot, Tak, 63110, Thailand \\ ${ }^{2}$ Microbiology and Immunology, University of Otago, Dunedin, Otago, 9016, New Zealand \\ ${ }^{3}$ School of Biological Sciences, Nanyang Technological University, Singapore, 637551, Singapore \\ ${ }^{4}$ Singapore Immunology Network, A*STAR, Singapore, 138648, Singapore \\ ${ }^{5} A *$ STAR ID Labs, A*STAR, Singapore, 138648, Singapore \\ ${ }^{6}$ Microbiology and Immunology, Yong Loo Lin School of Medicine, National University of Singapore, Singapore, 117545, Singapore \\ ${ }^{7}$ Centre for Tropical Medicine and Global Health, Nuffield Department of Medicine, University of Oxford, Oxford, UK
}

V1 First published: 22 Feb 2021, 6:39

https://doi.org/10.12688/wellcomeopenres.16564.1

Second version: 04 Aug 2021, 6:39

https://doi.org/10.12688/wellcomeopenres.16564.2

Latest published: $01 \mathrm{Apr}$ 2022, 6:39

https://doi.org/10.12688/wellcomeopenres.16564.3

\section{Abstract}

Background: Targeted malaria elimination strategies require highly sensitive tests to detect low density malaria infections (LDMI). Commonly used methods for malaria diagnosis such as light microscopy and antigen-based rapid diagnostic tests (RDTs) are not sensitive enough for reliable identification of infections with parasitaemia below 200 parasites per milliliter of blood. While targeted malaria elimination efforts on the Thailand-Myanmar border have successfully used high sample volume ultrasensitive quantitative PCR (UPCR) to determine malaria prevalence, the necessity for venous collection and processing of large quantities of patient blood limits the widespread tractability of this method.

Methods: Here we evaluated a real-time reverse transcription PCR (RT-PCR) method that reduces the required sample volume compared to UPCR. To do this, 304 samples collected from an active case detection program in Kayin state, Myanmar were compared using UPCR and RT-PCR.

Results: Plasmodium spp. RT-PCR confirmed 18 of 21 UPCR Plasmodium falciparum positives, while $P$. falciparum specific RT-PCR confirmed 17 of the 21 uPCR $P$. falciparum positives. Combining both RT-PCR results increased the sensitivity to $100 \%$ and specificity was $95.1 \%$.

Conclusion: Malaria detection in areas of low transmission and LDMI can benefit from the increased sensitivity of ribosomal RNA detection

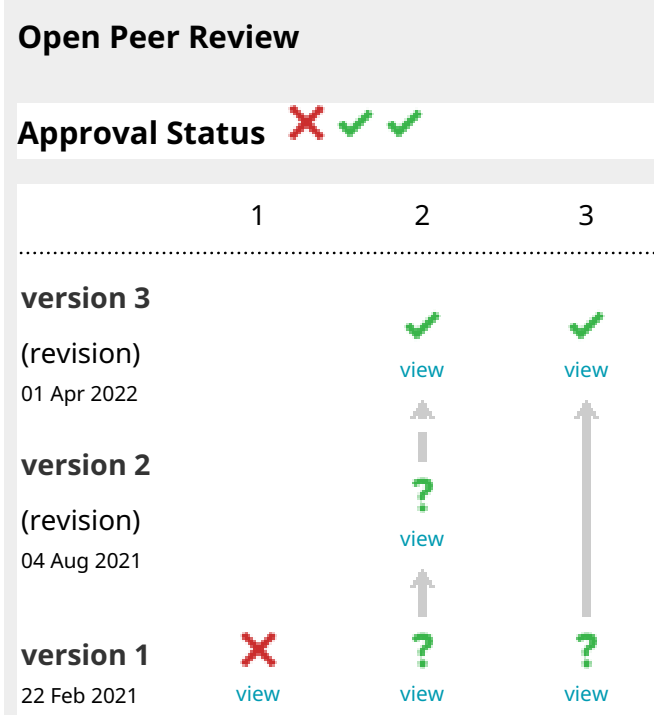

1. Gisely Melo, Instituto de Pesquisa Clínica

Carlos Borborema, Manaus, Brazil

2. Muneaki Hashimoto, National Institute of Advanced Industrial Science and Technology (AIST), Hayashi-cho, Japan

3. Julian Tanner (ID) The University of Hong Kong, Pokfulam, Hong Kong

Any reports and responses or comments on the 
by RT-PCR, especially where sample volume is limited. Isolation of high quality RNA also allows for downstream analysis of malaria transcripts.

\section{Keywords}

Low density malaria infection, Plasmodium, quantitative PCR, diagnostic, reverse transcription PCR (RT-PCR)

This article is included in the Mahidol Oxford

wellcometrus

MORU

Tropical Medicine Research Unit (MORU)

gateway. article can be found at the end of the article.

\section{Corresponding author: Peter Christensen (pr_christensen@hotmail.com)}

Author roles: Christensen P: Conceptualization, Data Curation, Formal Analysis, Methodology, Writing - Original Draft Preparation, Writing - Review \& Editing; Bozdech Z: Conceptualization, Methodology, Resources, Writing - Review \& Editing; Watthanaworawit W: Formal Analysis, Methodology, Writing - Review \& Editing; Rénia L: Resources, Writing - Review \& Editing; Malleret B: Resources, Writing - Review \& Editing; Ling C: Conceptualization, Formal Analysis, Supervision, Writing - Review \& Editing; Nosten F:

Conceptualization, Funding Acquisition, Resources, Writing - Review \& Editing

Competing interests: No competing interests were disclosed.

Grant information: This study was supported by the Wellcome Trust through a strategic award "Eliminating malaria to counter artemisinin resistance" [101148, https://doi.org/10.35802/101148]. Funding was also obtained from the following sources; the Bill and Melinda Gates Foundation; The Singapore Immunology Network, A*STAR core fund; the NUHS start-up funding [NUHSRO/2018/006/SU/01]; NUHS seed fund [NUHSRO/2018/094/T1]; the Wellcome Trust Mahidol University Oxford Tropical Medicine Research Programme and the New Zealand HRC eASIA [17/678] project grant.

The funders had no role in study design, data collection and analysis, decision to publish, or preparation of the manuscript.

Copyright: $\odot 2021$ Christensen P et al. This is an open access article distributed under the terms of the Creative Commons Attribution License, which permits unrestricted use, distribution, and reproduction in any medium, provided the original work is properly cited.

How to cite this article: Christensen P, Bozdech Z, Watthanaworawit W et al. Reverse transcription PCR to detect low density malaria infections [version 1; peer review: 2 approved with reservations, 1 not approved] Wellcome Open Research 2021, 6:39 https://doi.org/10.12688/wellcomeopenres.16564.1

First published: 22 Feb 2021, 6:39 https://doi.org/10.12688/wellcomeopenres.16564.1 


\section{Background}

As malaria burden reduces globally, the international community is working toward its elimination. Successful targeted malaria elimination strategies will require increased surveillance and highly sensitive tests capable of detecting asymptomatic and low density malaria infection (LDMI). These infections are often well below 200 parasites per milliliter and are an important disease reservoir capable of transmitting malaria that must be detected and eliminated ${ }^{1,2}$. Light microscopy and antigen based rapid diagnostic tests (RDTs) are the most common tests used in malaria prevalence surveys, and usually assess $5 \mu \mathrm{L}$ of whole blood per test, a volume which precludes reliable detection of LDMI. Ultrasensitive RDTs improve detection sensitivity of patients with a parasitaemia between 200 parasites $/ \mathrm{mL}$ and 10,000 parasites $/ \mathrm{mL}^{3}$, but are still limited by their low input volume. Molecular methods using the polymerase chain reaction (PCR) remain the only common and reliable method to detect LDMI. The sensitivity of PCR is due to its ability to detect single, specific nucleic acid molecules and use of concentrated DNA from a large sample. Widespread use of PCR based assays, namely real-time quantitative PCR (qPCR) and reverse-transcription PCR (RT-PCR), have revealed a new landscape of malaria prevalence particularly in low transmission areas ${ }^{4,5}$.

The targeted malaria elimination project (TME) on the Eastern Myanmar border used a high blood volume ultrasensitive qPCR (uPCR) to consistently detect parasitaemia down to 22 parasites per $\mathrm{mL}^{6}$, and revealed a high proportion of $\mathrm{LDMI}^{7}$. Major features of uPCR are its 7 copies of gene target, the high volume of blood tested and the ability to accurately quantify low density parasitaemia. Although increasing the blood volume of a PCR leads to higher sensitivity ${ }^{6}$, the collection of large numbers of high volume samples have their own specific limitations. These can include, the ethics approval required for venous blood draw, sample logistics, increased nucleic acid extraction cost and increased sample processing time. Another way to increase the sensitivity of PCR is to increase the number of specific nucleic acid targets per parasite by targeting specific RNA and DNA using RT-PCR. As previously reported by Kamau et al. $^{8}$, the primer set used in uPCR can be made more sensitive by incorporating reverse transcription prior to qPCR, enabling detection of the 7 genes encoding Plasmodium 18S ribosomal nucleic acid (rRNA) and its rRNA transcripts.

In this study, we compare the sensitivity and specificity of high sample volume, low target copy number ultrasensitive qPCRs, with reduced sample volume, high target copy number RT-PCR for the detection and quantification of Plasmodium spp. and $P$. falciparum.

\section{Methods}

We selected 304 samples with previously reported uPCR results: $21 P$. falciparum positive and 283 Plasmodium spp. negative for comparison to RT-PCR with increased target numbers per parasite but $30 \%$ of the sample volume.
Study area and sample collection

Active case detection samples were collected from rural Eastern Kayin (Karen) state of Myanmar between 2014 and 2015 as part of an international malaria elimination project. Full methods have been published ${ }^{9}$, briefly, $3 \mathrm{ml}$ of blood was drawn into an EDTA container from each adult, and transported on ice to the Shoklo Malaria Research Unit in Mae Sot, Tak, Thailand. Within 48 hours the samples were processed, and two aliquots of up to $500 \mu \mathrm{L}$ of packed red blood cells (PRBC) were stored at $-80^{\circ} \mathrm{C}$. Only $500 \mu \mathrm{L}$ samples were accepted for Malaria Elimination Task Force (METF) qPCR while RT-PCR included samples with at least $150 \mu \mathrm{L}$.

\section{Ultrasensitive qPCR (UPCR)}

DNA was extracted from $500 \mu \mathrm{l}$ of cryopreserved PRBC using QIAamp DNA Blood Midi Kit (Cat. No. 51185, Qiagen ${ }^{\mathrm{TM}}$ ) according to manufacturer's instructions. The DNA template was then dried in a vacuum concentrator, re-suspended in $10 \mu \mathrm{L}$ of PCR grade water and stored at $-20^{\circ} \mathrm{C}$ prior to $\mathrm{qPCR}$. Separate uPCRs specific for Plasmodium spp., $P$. falciparum and P. vivax were performed over 3 years from 2013 as previously described ${ }^{6}$. Briefly, uPCR was done in $10 \mu \mathrm{L}$ reactions that contained $2 \mu \mathrm{L}$ of DNA template with 1x QuantiTect Multiplex PCR No ROX mastermix (Cat. No. 204743, Qiagen ${ }^{\mathrm{TM}}$ ), $0.4 \mu \mathrm{M}$ each primer, and $0.2 \mu \mathrm{M}$ Taqman probe. Thermal cycling and signal acquisition was done on an ABI 7500 Fast real-time PCR machine with initial denaturation and enzyme activation at $95^{\circ} \mathrm{C}$ for $15 \mathrm{~min}$, then 50 cycles of denaturation at $94^{\circ} \mathrm{C}$ for $15 \mathrm{sec}$ followed by annealing and extension at $60^{\circ} \mathrm{C}$ for $60 \mathrm{sec}$. A reaction with exponential signal increase before cycle 40 was considered positive.

\section{Sample selection, nucleic acid extraction and RT-PCR}

Within 3 years of sample storage at $-80^{\circ} \mathrm{C}$, nucleic acid extraction and RT-PCR assays were performed on the second aliquot of PRBC for selected samples: 21 uPCR $P$. falciparum positives and $283 \mathrm{uPCR}$ negatives.

Nucleic acid was extracted using a Zymo Research Quick-RNA Miniprep (Plus) kit (Cat. No. R1058, Zymo Research ${ }^{\mathrm{TM}}$ ) from. Manufacturer's instructions for whole blood were followed with minor changes. These include, extraction from $150 \mu \mathrm{L}$ of white blood cell depleted PRBC in phosphate buffered saline (PBS) up to $200 \mu \mathrm{L}$ instead of $200 \mu \mathrm{l}$ of whole blood, DNase enzyme wasn't used, and RNA was eluted in $20 \mu \mathrm{L}$ of molecular grade water. Two RT-PCRs were performed on each extract in duplicate: the Plasmodium spp. specific assay using the same primer and probe set as UPCR, and a $P$. falciparum specific set targeting the DNA of the A-18S rRNA genes and its rRNA transcripts ${ }^{5}$. Both reactions had a final volume of $15 \mu \mathrm{L}$ and contained $4 \mu \mathrm{L}$ of RNA template, $1 \mathrm{x}$ Superscript III One-Step RT-PCR System master mix (Cat. No. 12574018, ThermoFisher Scientific ${ }^{\mathrm{TM}}$ ), $0.4 \mu \mathrm{M}$ forward and reverse oligo primer and $0.2 \mu \mathrm{M}$ of MGB Taqman probe. Amplification and signal acquisition were done on an ABI 7500 Fast real-time PCR machine with cycling conditions as follows: reverse 
transcription at $45^{\circ} \mathrm{C}$ for $30 \mathrm{~min}$, enzyme activation at $95^{\circ} \mathrm{C}$ for $2 \mathrm{~min}$, followed by 50 cycles of denaturation at $95^{\circ} \mathrm{C}$ for $15 \mathrm{sec}$ and combined annealing and extension steps at $60^{\circ} \mathrm{C}$ for $60 \mathrm{sec}$. A reaction with exponential signal increase before cycle 40 was considered positive.

\section{Primer and probe sequences}

Plasmodium spp. specific primer and probe sequences used by uPCR and RT-PCR were published previously by kamau et $a{ }^{8}{ }^{8}$. The forward primer sequence was 5'-GCTCTTTCTTGATTTCTTGGATG-3', reverse primer was 5'-AGCAGGTTAAGATCTCGTTCG-3' and the probe sequence was 5'-ATGGCCGTTTTTAGTTCGTG-3' labeled with FAM as reporter and a TAMRA quencher. The primer and probe sequences used to identify $P$. falciparum infection in uPCR positive samples were published by Perandin et al. ${ }^{10}$. The forward primer was 5'-CTTTTGAGAGGTTTTGTTACTTTGAGTAA-3', reverse was 5'-TATTCCATGCTGTAGTATTCAAACACAA-3' and the probe sequence was 5'-TGTTCATAACAGACGGGTAGTCATGATTGAGTTCA-3' with FAM and TAMRA as reporter and quencher respectively. RT-PCR primer sets were taken from the publication by Sumari et al. ${ }^{5}$. The forward primer was 5'-TCCGATAACGAACGAGATCTTAAC-3', reverse primer was 5'-ATTATAGTTACCTATGTTCAATTTCA-3' and the minor groove binding probe sequence was 5'-TAGCGGCGAGTACACTATA-3' labelled with a FAM reporter and BHQ1 quencher.

\section{Standard curve}

Standard reference curves for the RT-PCR and UPCR were made using aliquots of 10,000 flow cytometry sorted small ring stage $P$. falciparum (3D7) parasites ${ }^{11}$. The method used to extract the nucleic acids from these parasites depended on the assay used (RT-PCR or uPCR). For the uPCR standard curve, Qiagen DNA Blood Mini Kit (Cat. No. 51106, Qiagen) was used to extract DNA, this was eluted in $200 \mu \mathrm{L}$ of sterile water, dried in a partial vacuum at $30^{\circ} \mathrm{C}$ for consistency with sample extraction, and re-suspended in $200 \mu \mathrm{L}$ of Qiagen AE buffer ${ }^{6}$. Nucleic acid for the RT-PCR standard curve was extracted using the Zymo Quick-RNA Miniprep (Plus) (Cat. No. R1058, Zymo Research) kit as above but eluted in $40 \mu \mathrm{L}$ of water. Serial one in five dilutions were made with these extracts to make 7 standards. The uPCR standard curve ranged from 100 to 0.032 parasites per qPCR reaction and the RT-PCR standard curve ranged from 1000 parasites to 0.064 parasites per reaction.

\section{Analysis}

Plasmodium spp. and P. falciparum diagnostic accuracy was calculated by comparing RT-PCR results to the uPCR result in $2 \times 2$ cross tabulation with uPCR as gold standard in STATA version 15.1 (StataCorp LLC, USA). Results of both RT-PCRs were also combined and compared to UPCR, where a positive test by at least one RT-PCR was considered positive. Continuous non-parametric data were compared with Kruskal-Wallis equality-of-populations rank test. $P$ values $<0.05$ were considered statistically significant. Analyses were performed using STATA version 15.1. Agreement between the two Plasmodium specific PCR quantifications (spp RT-PCR and uPCR) was determined using Bland-Altman analysis of log transformed data (difference vs average) using GraphPad Prism version 6.07 for Windows, GraphPad Software (La Jolla California USA).

\section{Results}

Plasmodium spp. RT-PCR

Plasmodium spp. RT-PCR confirmed 18 of 21 Plasmodium spp. uPCR positives and an additional 9 positive reactions from the 283 uPCR negatives. The sensitivity and specificity of Plasmodium spp. RT-PCR was $85.7 \%$ and $96.8 \%$ respectively when compared to uPCR (Table $1^{13}$ ). The positive predictive value (PPV) of this test was $66.7 \%$ with a $95 \%$ confidence interval (CI) of $46-83.5 \%$ and negative predictive value (NPV) was $98.9 \%$ with a $95 \%$ CI of $96.9-99.8 \%$.

\section{P. falciparum RT-PCR}

Using the $P$. falciparum specific RT-PCR, 16 of the 21 uPCR $P$. falciparum positives were confirmed along with 5 extra positives from the 283 negatives. Sensitivity and specificity of this test was $76.2 \%$ and $98.2 \%$ respectively when compared to uPCR (Table $1^{13}$ ). PPV for this PCR was $76.2 \%$ (95\% CI $=52.8$ $-91.8 \%)$ and NPV was $98.2 \%(95 \% \mathrm{CI}=95.9-99.4 \%)$.

Combined Plasmodium spp. and P. falciparum RT-PCR Combining the results of both Plasmodium spp. and P. falciparum RT-PCRs, all $21 \mathrm{uPCR}$ positives are confirmed positive (100\% Sensitivity) with 14 additional/false positives (specificity 95.1\%) (Table $1^{13}$ ). These combined results gave

Table 1. Results of three PCRs on 304 malaria survey samples: tabulated results from Plasmodium spp. uPCR, Plasmodium spp. RT-qPCR and P. falciparum RT-qPCR including total positives, total negatives, false positives, false negatives, sensitivity and specificity using Plasmodium spp. UPCR results as gold standard.

\begin{tabular}{|l|c|c|c|c|c|c|}
\hline & Positive & Negative & False Positive & False Negative & Sensitivity (95\% CI) & Specificity (95\% CI) \\
\hline Plasmodium spp. uPCR & 21 & 283 & N/A & N/A & N/A & N/A \\
\hline Plasmodium spp. RT-PCR & 27 & 277 & 9 & 3 & $85.7 \%(63.7-97 \%)$ & $96.8 \%(94-98.5 \%)$ \\
\hline P. falciparum RT-PCR & 21 & 283 & 5 & 5 & $76.2 \%(52.8-91.8 \%)$ & $98.2 \%(95.9-99.4 \%)$ \\
\hline Combined RT-PCR & 35 & 269 & 14 & 0 & $100 \%(83.9-100 \%)$ & $95.1 \%(91.8-97.3 \%)$ \\
\hline
\end{tabular}


a PPV of $60 \%(95 \% \mathrm{CI}=42.1-76.1 \%)$ and a NPV of $100 \%$ $(95 \% \mathrm{CI}=98.6-100 \%)$.

\section{Quantification}

No significant differences were found for the calculated parasites $/ \mathrm{ml}(P$ 0.5506) for the three different methods with geometric means of $2.78 \mathrm{E} \times 10^{4}\left(95 \%\right.$ CI $\left.4.34 \times 10^{3}-1.79 \times 10^{5}\right)$, $1.81 \times 10^{4}\left(1.35 \times 10^{3}-2.41 \times 10^{5}\right)$ and $6.85 \mathrm{E} \times 10^{3}\left(1.09 \times 10^{3}\right.$ $-4.31 \times 10^{4}$ ) for the Plasmodium spp. RT-qPCR, P. falciparum
RT-qPCR and the Plasmodium spp. uPCR respectively (Figure 1).

Bland-Altman analysis of log transformed parasitaemia data did not show any significant systematic bias between uPCR and RT-PCR measurements (Bias $=-0.3786+/$ - S.D 1.948), $95 \%$ of our differences fall between 2 standard deviations or more precisely $1.96 \times$ S.D $(1.948)=3.82$. This implies a reasonable amount of agreement between uPCR and RT-PCR.

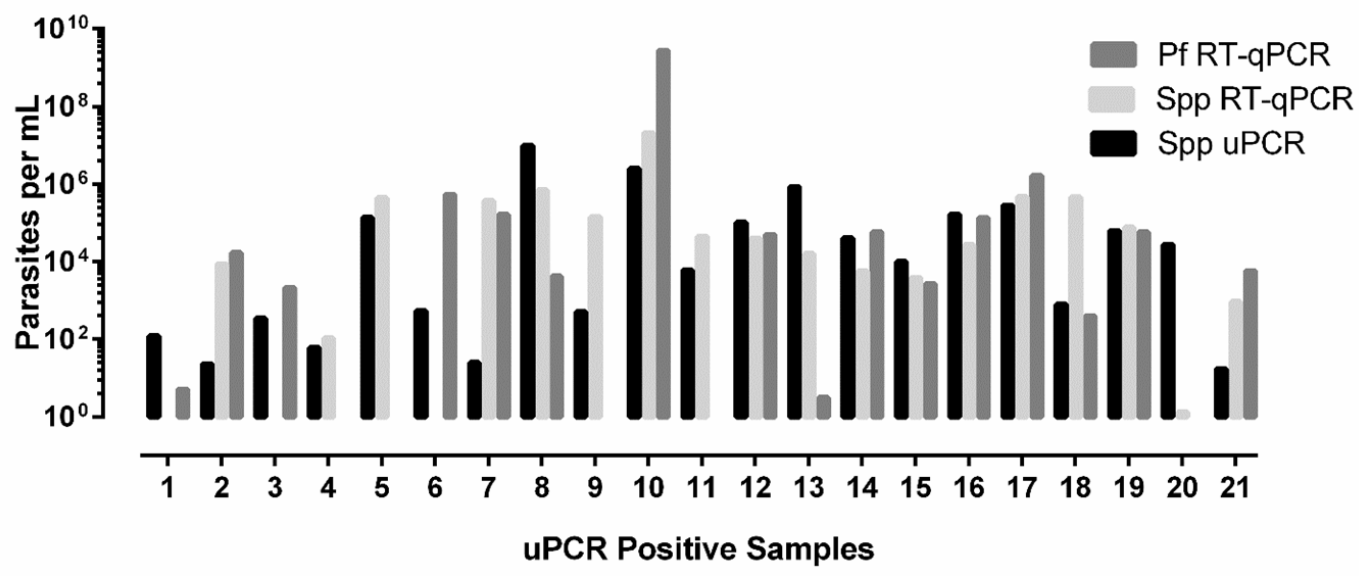

Figure 1. Parasite quantification by Plasmodium species ultrasensitive PCR (spp uPCR), Plasmodium species RT-qPCR (Spp RT-qPCR) and Plasmodium falciparum RT-qPCR (Pf RT-qPCR). Quantification of parasitaemia from three PCRs on 21 uPCR positives from the TME malaria survey of Kayin state, Myanmar.

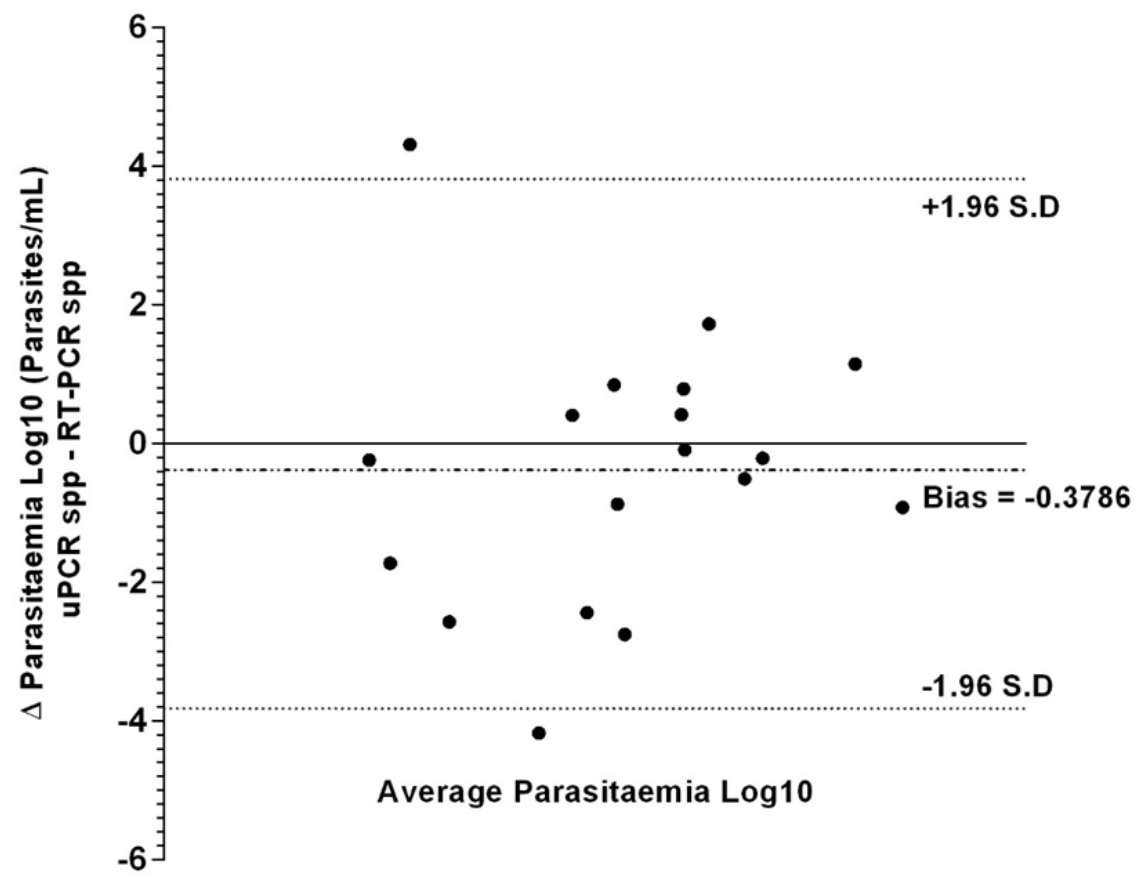

Figure 2. Bland-Altman analysis (difference vs average) of uPCR and RT-PCR quantification data from the TME malaria survey of Kayin state, Myanmar. 


\section{Discussion}

LDMI detection is essential for effective targeted elimination programs, necessitating the careful selection of a detection assay that is appropriate to the setting and study requirements. Sample volume, storage conditions and transit time are important factors, as well as the required assay sensitivity, specificity and its cost. RNA is generally less stable than DNA, and accurate RNA quantification often requires normalization due to variable transcription rates. It is for these reasons that DNA based qPCR was chosen for the original study as this approach enabled accurate quantification of LDMI in a setting where samples from remote locations would likely experience delays.

The targeted malaria elimination (TME) project on the Eastern Myanmar border used conserved regions of the $18 \mathrm{~S}$ rRNA genes as the target for qPCR. This high sample volume uPCR assay was modified from the RT-PCR published by Kamau et $a l .{ }^{8}$ for their detection of low density malaria infections (LDMI). After uPCR detected a high prevalence of LDMI in the region ${ }^{9}$, and with continued surveillance in mind, we wanted a lower sample volume assay with similar LDMI detection sensitivity. As Kamau et al. has shown, using this primer set as a RT-PCR significantly increases the sensitivity of the assay. The increased sensitivity of RT-PCR is due to the increased number of targets per parasite (compared to uPCR). These amplification targets include the 18S rRNA genes, and the structural RNA of each ribosome. Plasmodium genus specific uPCR amplifies type A and S 18S ribosomal RNA genes distributed on chromosomes $1,5,7,11$ and 13. A positive qPCR reaction requires at least one of these genes to be included in the assay. Alternatively, RT-PCR can detect these gene loci, and their gene transcripts (rRNA). The increased target copy number per parasite means a smaller fraction of parasite is needed to provide a positive reaction, leading to less false negatives and an opportunity for further downstream applications. The downside to increased sensitivity and target copy is the variable nature of gene expression and relative fragility of RNA molecules. In general this makes accurate quantification of parasitaemia by RT-PCR more challenging, and is one of the reasons for outliers in our inter-assay quantification comparison. In future studies the use of a comprehensive methodology tailored specifically to RNA preservation, extraction and amplification would improve sensitivity and allow for downstream applications involving whole-transcriptome profiling important for studies on transmission, pathogenesis and virulence ${ }^{12}$.

While there were obvious differences in quantification for some samples by each method, these did not reach statistical significance. One reason for these outliers was the use of different stored aliquot volumes between assay types. The uPCR aliquot was fixed at $500 \mu \mathrm{L}$ while the second aliquot, which was used for RT-PCR ranged from $150 \mu \mathrm{L}$ to $1 \mathrm{~mL}$ prior to freezing. Because RT-PCR targeting rRNA is capable of detecting tiny fractions of a single parasite, the original lysed sample volume becomes an important detail. Assuming a single freeze thaw lyses blood stage Plasmodium and a Plasmodium assay has a hundred thousand targets per parasite, then a single lysed parasite divided into one hundred aliquots can produce one hundred positive reactions, if the parasite wasn't lysed beforehand then only 1 of 100 would be positive. This can lead to people describing their assay sensitivity well below the sample volume used, a theoretical impossibility unless detecting free circulating nucleic acids outside of parasite cells.

Alternatively, LDMI detection relying on DNA, will have a significant reduction in sensitivity if only a fraction of the DNA template is tested. This is exemplified by the lowest concentration standard used for quantification in uPCR. This standard theoretically contains 0.032 parasites per PCR reaction, at this concentration there is a 1 in 5 chance for the reaction to be positive $(7$ target genes per parasite $\times 0.032=0.2$ copies per reaction). These factors need to be considered when setting up a qPCR standard curve for LDMI quantification. A reliable standard curve for $\mathrm{qPCR}$ requires at least one copy of its target at the lowest concentration. In conclusion, the success of any LDMI detection protocol relies on the careful consideration of the following factors: sample volume, elution volume, template volume per assay reaction, primer set target and assay type (uPCR or RT-PCR).

\section{Conclusion}

Our experience of the different types of assay suggest that for a LDMI program requiring highly sensitive, accurate quantification and where venous blood collection is possible, uPCR is recommended. In an environment where blood volume is limited (i.e. finger prick sampling) and quantification accuracy of parasitaemia is less important, RT-PCR is a suitable alternative.

\section{Declaration}

\section{Ethics approval and consent to participate}

First, community engagement teams sought community approval ahead of the survey date. Then, survey participants received individual information in their language, and informed consent was obtained from each individual before they provided a venous blood sample. Appropriate treatment for Plasmodium falciparum or Plasmodium vivax was available for all RDT-positive individuals.

The METF project has ethical approval from the Lower Myanmar Department of Medical Research Ethics' committee (reference 73/ETHICS2014, dated 25 November 2014, and renewed in November 2015 and 2016 under the same reference).

\section{Data availability}

\section{Underlying data}

Open Science Framework: Reverse transcription PCR to detect low density malaria infections. https://doi.org/10.17605/OSF. $\mathrm{IO} / 76 \mathrm{~K} 9 \mathrm{R}^{13}$

This project contains the following underlying data:

- Plasmodium quantifications_Parasites per mL.xls (Contains Plasmodium spp., P. falciparum quantifications (parasite per $\mathrm{mL}$ whole blood) and the threshold cycle for all RT-PCRs) 
Data are available under the terms of the Creative Commons Zero "No rights reserved" data waiver (CC0 1.0 Public domain dedication).

\section{Acknowledgements}

The authors would like to thank all the staff and participants involved in the malaria elimination project.
1. Sturrock HJW, Hsiang MS, Cohen JM, et al.: Targeting asymptomatic malaria infections: active surveillance in control and elimination. PLOS Med. 2013; 10(6): e1001467.

PubMed Abstract | Publisher Full Text | Free Full Text

2. Okell LC, Bousema T, Griffin JT, et al.: Factors determining the occurrence of submicroscopic malaria infections and their relevance for control. Nat Commun. 2012; 3: 1237

PubMed Abstract | Publisher Full Text | Free Full Text

3. Landier J, Haohankhunnatham W, Das S, et al.: Operational Performance of a Plasmodium falciparum Ultrasensitive Rapid Diagnostic Test for Detection of Asymptomatic Infections in Eastern Myanmar. J Clin Microbiol. 2018; 56(8): e00565-18.

PubMed Abstract | Publisher Full Text | Free Full Text

4. Parker DM, Landier J, Thu AM, et al.: Scale up of a Plasmodium falciparum elimination program and surveillance system in Kayin State, Myanmar [version 2; peer review: 2 approved]. Wellcome Open Res. 2017; 2: 98. PubMed Abstract | Publisher Full Text | Free Full Text

5. Sumari D, Mugasa J, Selemani M, et al.: Prevalence of submicroscopic Plasmodium falciparum infections in asymptomatic children in low transmission settings in Bagamoyo, Tanzania. MWJ. 2016; 7. Reference Source

6. Imwong $\mathrm{M}$, Hanchana $\mathrm{S}$, Malleret $\mathrm{B}$, et al:: High-throughput ultrasensitive molecular techniques for quantifying low-density malaria parasitemias. J Clin Microbiol. 2014; 52(9): 3303-9.

PubMed Abstract | Publisher Full Text | Free Full Text

7. von Seidlein L, Peto TJ, Landier J, et al.: The impact of targeted malaria elimination with mass drug administrations on falciparum malaria in
Southeast Asia: A cluster randomised trial. PLoS Med. 2019; 16(2): e1002745. PubMed Abstract | Publisher Full Text | Free Full Text

8. Kamau E, Tolbert LS, Kortepeter L, et al.: Development of a highly sensitive genus-specific quantitative reverse transcriptase real-time PCR assay for detection and quantitation of plasmodium by amplifying RNA and DNA of the 18S rRNA genes. J Clin Microbiol. 2011; 49(8): 2946-53.

the 18S rRNA genes. J Clin Microbiol. 2011; 49(8): 2946-53.
PubMed Abstract | Publisher Full Text | Free Full Text

9. Landier J, Kajeechiwa L, Thwin MM, et al.: Safety and effectiveness of mass drug administration to accelerate elimination of artemisinin-resistant falciparum malaria: A pilot trial in four villages of Eastern Myanmar [version 1; peer review: 2 approved]. Wellcome Open Res. 2017; 2: 81. PubMed Abstract | Publisher Full Text | Free Full Text

10. Perandin F, Manca N, Calderaro A, et al.: Development of a real-time PCR assay for detection of Plasmodium falciparum, Plasmodium vivax, and Plasmodium ovale for routine clinical diagnosis. J Clin Microbiol. 2004; 42(3): 1214-9.

PubMed Abstract | Publisher Full Text | Free Full Text

11. Malleret B, Claser C, Ong ASM, et al:: A rapid and robust tri-color flow cytometry assay for monitoring malaria parasite development. Sci Rep. 2011; 1: 118. PubMed Abstract | Publisher Full Text | Free Full Text

12. Kucharski M, Tripathi J, Nayak S, et al.: A comprehensive RNA handling and transcriptomics guide for high-throughput processing of Plasmodium blood-stage samples. Malar J. 2020; 19(1): 363.

PubMed Abstract | Publisher Full Text | Free Full Text

13. Christensen $P$, Nosten F, Bozdech Z, et al.: Reverse transcription PCR to detect low density malaria infections. 2021.

http://www.doi.org/10.17605/OSF.IO/76K9R 


\section{Open Peer Review}

\section{Current Peer Review Status: $\mathrm{X}$ ? ?}

\section{Version 1}

Reviewer Report 22 July 2021

https://doi.org/10.21956/wellcomeopenres.18253.r44476

(C) 2021 Tanner J. This is an open access peer review report distributed under the terms of the Creative Commons Attribution License, which permits unrestricted use, distribution, and reproduction in any medium, provided the original work is properly cited.

\section{Julian Tanner}

School of Biomedical Sciences, Li Ka Shing Faculty of Medicine, The University of Hong Kong, Pokfulam, Hong Kong

In this article, the authors present data on using RT-PCR on small volume clinical samples.

This is a fairly simple, although logistically challenging, study, particularly given the present COVID situation. The study adds value to the literature around this topic and given this is:

1. An important topic.

2. A journal which accepts submissions Wellcome Open Research without concerning too much with impact

3. Adds value for those looking at PCR methods in malaria-endemic regions.

4. Has suitable ethical approvals.

I would support the indexing of this article.

Some comments to improve:

1. Figure 1 are these data in triplicate, can error bars be added?

2. Figure 2 besides Bland-Altman can further analysis be added to add some more depth to the paper?

Is the work clearly and accurately presented and does it cite the current literature? Yes

Is the study design appropriate and is the work technically sound? Yes 
Are sufficient details of methods and analysis provided to allow replication by others? Yes

If applicable, is the statistical analysis and its interpretation appropriate? Yes

Are all the source data underlying the results available to ensure full reproducibility? Yes

Are the conclusions drawn adequately supported by the results? Yes

Competing Interests: No competing interests were disclosed.

Reviewer Expertise: Molecular diagnostics.

I confirm that I have read this submission and believe that I have an appropriate level of expertise to confirm that it is of an acceptable scientific standard, however I have significant reservations, as outlined above.

Author Response 27 Jul 2021

Peter Christensen, Mahidol University, Maesot, Thailand

Dear Reviewer, thanks for you time reviewing our paper.

I have responded to your questions below.

1. Figure 1 are these data in triplicate, can error bars be added?

2. Unfortunately not. This is one reason we called it RT-PCR not RT-qPCR (the other is a lack of internal control). We were trying to add as much concentrated sample into the reaction as possible to have detection sensitivity similar to UPCR. This was the catch, we are forced to use as much sample as possible per reaction to achieve high sensitivity, but we don't want the logistics issues of large sample volumes. So we had to decide; either we decrease the assay sensitivity to increase its quantification accuracy, or put the whole sample into the PCR reaction. As this PCR will be used for 'Hot Spot' detection at the village level, we prioritized high sensitivity over high precision.

3. Figure 2 besides Bland-Altman can further analysis be added to add some more depth to the paper?

4. I'm hesitant to analyse the quantifications further as they're not high quality (not triplicate, not normalised) and accurate quantification of LDMI is not particularly important (low detection limit is more important).

5. I'll be adding microscopy and RDT results to the next draft and more information on the species present. Some of these samples were mixed Pf+Pv infections.

I hope I have answered your questions fully, The next draft will be out soon. 


\section{Best Regards,} Peter C

Competing Interests: No competing interests were disclosed.

Reviewer Report 25 June 2021

https://doi.org/10.21956/wellcomeopenres.18253.r44495

(C) 2021 Hashimoto $\mathbf{M}$. This is an open access peer review report distributed under the terms of the Creative Commons Attribution License, which permits unrestricted use, distribution, and reproduction in any medium, provided the original work is properly cited.

\section{Muneaki Hashimoto}

Health Research Institute, National Institute of Advanced Industrial Science and Technology (AIST), Hayashi-cho, Takamatsu, 761-0395, Japan

In this study, the authors compared RT-qPCR with uPCR to develop ultra-sensitive and quantitative Plasmodium parasite detection methods. Previously, Kamau et al. showed that the detection limit of RT-qPCR is significantly lower than that of UPCR in experiments with in vitro cultured parasites. Using RT-qPCR, the current study investigated the ability to detect the parasites with ultrasensitivity in real-patient blood samples and examined the possibility of reducing the required blood sample volume. The authors showed that parasitemia calculated by spp. UPCR was not statistically significantly different from that calculated by Pf RT-qPCR and spp. RT-qPCR, respectively, concluding that RT-qPCR may be an alternative to UPCR.

My comments are below:

1. In this study, UPCR was performed as the gold standard method. The gold standard method should have sufficiently high accuracy when compared with the method undergoing evaluation. What is the rationale for UPCR being the gold standard when assessing RTqPCR?

2. What is the cause of the false-positive results detected by RT-qPCR? For example, is there any risk of detecting rRNA of other pathogens by performing RT-qPCR? It is important to evaluate whether this method is suitable for actual blood samples

3. In the paper, what should be written as RT-qPCR was written as RT-PCR. The authors need to revise this.

Is the work clearly and accurately presented and does it cite the current literature? Yes

Is the study design appropriate and is the work technically sound? Partly 
Are sufficient details of methods and analysis provided to allow replication by others? Yes

If applicable, is the statistical analysis and its interpretation appropriate? I cannot comment. A qualified statistician is required.

Are all the source data underlying the results available to ensure full reproducibility? Yes

Are the conclusions drawn adequately supported by the results? Partly

Competing Interests: No competing interests were disclosed.

Reviewer Expertise: Development of malaria diagnosis devices

I confirm that I have read this submission and believe that I have an appropriate level of expertise to confirm that it is of an acceptable scientific standard, however I have significant reservations, as outlined above.

Author Response 27 Jul 2021

Peter Christensen, Mahidol University, Maesot, Thailand

Dear Reviewer,

Thank you for your time reviewing the paper. It is very much appreciated.I have replied to each of your questions below in bold.

1. In this study, UPCR was performed as the gold standard method. The gold standard method should have sufficiently high accuracy when compared with the method undergoing evaluation. What is the rationale for UPCR being the gold standard when assessing RT-qPCR?

2. We use UPCR because it has been fully evaluated for sensitivity and specificity in 'Imwong et al. JCM 2014' - 2500x more sensitive than microscopy (true gold standard) and 50x more sensitive than finger prick + PCR. Also, UPCR is a standard method at the SMRU and I had already produced UPCR results from these samples. My supervisor wanted to collect less sample without losing too much sensitivity, and I knew the primer set was more sensitive in RT-PCR format (Kamau et. al. JCM 2011). The samples had a lower volume cryopreserved second aliquot, so I tried the UPCR in RT-PCR format. Another reviewer has asked for the microscopy (true gold standard detection) and RDT results, which will be included in the next draft. Both are inappropriate for comparison to RT-PCR due to low sensitivity, which you'll see in the next draft.

3. What is the cause of the false-positive results detected by RT-qPCR? For example, is there any risk of detecting rRNA of other pathogens by performing RT-qPCR? It is important to evaluate whether this method is suitable for actual blood samples

4. The 18SrRNA primers are unique to Plasmodium, so false positives are either real positives or, more likely, contamination from the standard during plate 
preparation (false positive from uPCR (Imwong 2014) were found to be caused by standard contamination). Unfortunately, we were trying to include as much sample as possible to to increase sensitivity of the PCR, so we had no sample left over to investigate these false positives further.

5. In the paper, what should be written as RT-qPCR was written as RT-PCR. The authors need to revise this.

6. Our PCR doesn't meet the criteria for RT-qPCR so one of the authors wasn't happy naming it 'qPCR' (The PCR is not in triplicate, and doesn't have an internal control for normalising quantification). I understand the confusion, because we go on to compare quantification (which I assumed would be different but to my surprise wasn't). In my opinion the quantification of LDMI is less useful than the low limit of detection and could be removed entirely from the paper (especially without knowing the life stage - ring vs schizont will have vastly different ribosome quantities).

I hope this answers your questions, I'll have the second draft in soon.

Regards,

Peter C

Competing Interests: No competing interests were disclosed.

Reviewer Report 20 April 2021

https://doi.org/10.21956/wellcomeopenres.18253.r42783

(C) 2021 Melo G. This is an open access peer review report distributed under the terms of the Creative Commons Attribution License, which permits unrestricted use, distribution, and reproduction in any medium, provided the original work is properly cited.

\section{Gisely Melo}

Tropical Medicine Foundation Dr. Heitor Vieira Dourado, Instituto de Pesquisa Clínica Carlos Borborema, Manaus, Brazil

\footnotetext{
Abstract:

What was the target used? Was all PCR targeted to a specific gender? What was the gold standard used? The method is not clear. Why used only falciparum used for the manuscript?

Introduction:

What is the target? What is the comparison with methods used as the gold standard of PCR?

Methods:

Why was only Plasmodium falciparum used? What chance does the target fail to detect other malaria? Which parasitemia of the people studied to study low parasitemia? If used, was gold pattern used as a thick blood smear or RDT? The target for RT-PCR was for Plasmodium? Two RTPCRs were performed on each extract in duplicate: Plasmodium spp. and are they sensitive enough? Using UPCR as a gold standard would not be the most appropriate use of the thick blood
} 
smear as a control. It is not clear that he will use a method for gender and species for methodology.

\section{Results:}

Using UPCR as a gold standard is not the most suitable. How to be sure of positive positives and false negatives? Why was parasitemia not compared in the studied methods to verify the comparison between the methods to verify the smallest parasitemia that it would detect? In quantification, it is more appropriate to perform correlation analysis to compare a parasitemia between the methods.

\section{Discussion:}

The targets should have been described in the methodology as well. What are the limitations of the study?

Is the work clearly and accurately presented and does it cite the current literature? Partly

Is the study design appropriate and is the work technically sound?

No

Are sufficient details of methods and analysis provided to allow replication by others? Yes

If applicable, is the statistical analysis and its interpretation appropriate? Partly

Are all the source data underlying the results available to ensure full reproducibility? Yes

Are the conclusions drawn adequately supported by the results? Partly

Competing Interests: No competing interests were disclosed.

I confirm that I have read this submission and believe that I have an appropriate level of expertise to state that I do not consider it to be of an acceptable scientific standard, for reasons outlined above.

Author Response 22 Apr 2021

Peter Christensen, Mahidol University, Maesot, Thailand

Thank you for your time reviewing this paper, I appreciate the effort.

Hopefully I can address all your comments in the next draft.

Info on target \& sensitivity specifictity data: 
I will update the paper to include more information about the primer targets earlier and will add the previously published Sens and Spec for the Plasmodium spp. primer set used by UPCR and RT-PCR. It has been done in the original paper for both qPCR and qRT-PCR and then again for high volume UPCR. Will adding previously published data be satisfactory?

Why only falciparum:

We used Pf only because we can produce very young rings invitro that are then FACS sorted for a more consistent standard, and because clinical vivax samples often contain late stages (which may contain more than one nucleus per infected red cell and also contain more ribosomal material). Furthermore, although there is a growing realisation of vivax malaria's importance; the funder and clinicians at the field site still consider the sensitive and timely diagnosis of Pf a priority. Nonetheless I wish to assure the reviewer that we also would have preferred to have a Pv data set (esp. considering Pv is the most prevalent in the region); however due to the large proportion of sample per reaction (4ul of DNA template per rxn \& only 20ul DNA eluted) this means we can only do two PCRs in duplicate. This is a limitation of this type of high blood volume per reaction assay. I can discuss this limitation.

\section{Clarify targets:}

Only 2 new RT-PCR results were produced in this study, both target 18S RNA. One was Plasmodium genus specific, the other was $P$. falciparum specific. The original Pf classification given by uPCR used another Pf primer set from Perandin et al. JCM 2004.

In hindsight I should have used this same Pf primer set for my RT-PCR assays. The reason I used primers from Sumari et al. is because they had been used in RT-PCR format and we originally included gametocyte data using PfS25 primers from that paper as well. We didn't have enough sample to complete the data set, so it was removed from the paper.

\section{RDT and Microscopy:}

Microscopy would be better as it is the gold standard. But the LOD is so low it would not be able to detect the majority of samples in this data set (13 of 21 are submicroscopic $(<50,000$ $\mathrm{p} / \mathrm{mL})$ ). Unfortunately RDT data from the malaria elimination program, was found to have some important QA issues (the specific batch of PfHRP-2 RDTs provided by Global Fund)).

\section{Statistics:}

I thought correlation was appropriate here as well, but our statistician sent me this paper ( https://www.ncbi.nlm.nih.gov/pmc/articles/PMC4470095/). So I used bland-altman for assessing agreement (both similarities and differences) between test measurements. Originally I wanted this assay to be yes/no and semi-quantitative and expected the analysis to not show agreement between tests. The reason thought this is because Kamau et al have already shown this primer set is less accurate at low parasitaemia when used as qRT-PCR.

Number of LDMI:

I will add the number of samples that were low density (less than 50 parasites/uL) by uPCR result: 13 of 21 were LDMI.

Again we wish to thank the reviewer for their time and expertise, Regards, 
Peter C

Competing Interests: No competing interests were disclosed. 